\title{
Resource Scheduling Algorithms in Long Term Evolution (LTE)
}

\author{
Mohammed Alhadi Abduljalil \\ Communication Department, alneeline university Khartoum, Sudan
}

\begin{abstract}
LTE is the evolution of the Universal Mobile Telecommunications System (UMTS). LTE intends to create a new radio-access technology which will provide high data rates, a low latency and a greater spectral efficiency. The downlink physical resource of the LTE is represented as a time-frequency resource grid consisting of multiple Resource Blocks (RB). A scheduler is a key element in the Base Station (BS) and it assigns the time and frequency resources to different users in the cell. In this thesis the Best CQI and Round Robin scheduling algorithms were investigated. The implementation, analysis and comparison of both scheduling algorithms were done through simulations executed on a MATLAB-based downlink link level simulator from the Vienna University. The impact of the scheduling schemes on the throughput was examined and the fairness of each scheduling scheme was investigated.
\end{abstract}

Keywords: LTE, Radio Resource, Scheduling algorithms, Round Robin Scheduling (RR), Best Channel Quality Indicator Scheduling (Best CQI).

\section{Introduction}

Long Term Evolution (LTE) is a 4G wireless broadband technology developed by the Third Generation Partnership Project (3GPP), an industry trade group.3GPP engineers named the technology "Long Term Evolution" because it represents the next step (4G) in a progression from GSM, a $2 \mathrm{G}$ standard, to UMTS, the $3 \mathrm{G}$ technologies based upon GSM [2]. LTE provides an uplink speed of up to 50 megabits per second (Mbps) and a downlink speed of up to $100 \mathrm{Mbps}$, and a bandwidth scalable from $1.25 \mathrm{MHz}$ to $20 \mathrm{MHz}$ This suits the needs of different network operators that have different bandwidth allocations, and also allow operators to provide different services based on spectrum. LTE also improves the spectral efficiency in $3 \mathrm{G}$ networks, allowing carriers to provide more data and voice services over a given bandwidth [3].

LTE is designed to meet carrier needs for high-speed data and media transport as well as high-capacity voice support well into the next decade .It encompasses high-speed data, multimedia unicast and multimedia broadcast services. When LTE technology is rolled out by mobile broadband providers over their networks it will offer users a whole range of benefits and new facilities, which will allow them to get far more out of their mobile broadband service [3].

This paper presents two scheduling algorithms Round Robin Scheduling (RR) and the Best Channel Quality Indicator Scheduling (Best CQI) to share the available resources efficiently while trying not to interfere in high manner with the other transmissions and also desire is to improve the speed of transmissions.

\section{Over Veiw Of Scheduling Algorithms}

The radio resource management algorithms are important to optimize the system capacity and end user performance. The network algorithms are not standardized but the network vendors and operators can design and tune the algorithms according to the needs. There are several algorithms in LTE such as [13]:

\section{A. Round Robin Scheduling}

Round robin method is used to allocate the radio resources to users, the first user will be served with the whole frequency spectrum for a specific period of time and then serve the next user for another time period. The previously served user will be placed at the end of the waiting queue to be served again in the next round. The entire new resources requests will also be placed at the end of the waiting queue. This scheme offers great fairness in radio resource assignment among the users but with low throughput.

The principal advantage of Round Robin scheduling is the guaranty of fairness for all users. Furthermore Round Robin is easy to implement, that is the reason why it is usually used by many systems. Since, Round Robin does not take the channel quality information into consideration; it results in low user throughput. The flowchart of the Round Robin scheduling is shown in Fig.1 [3]. 


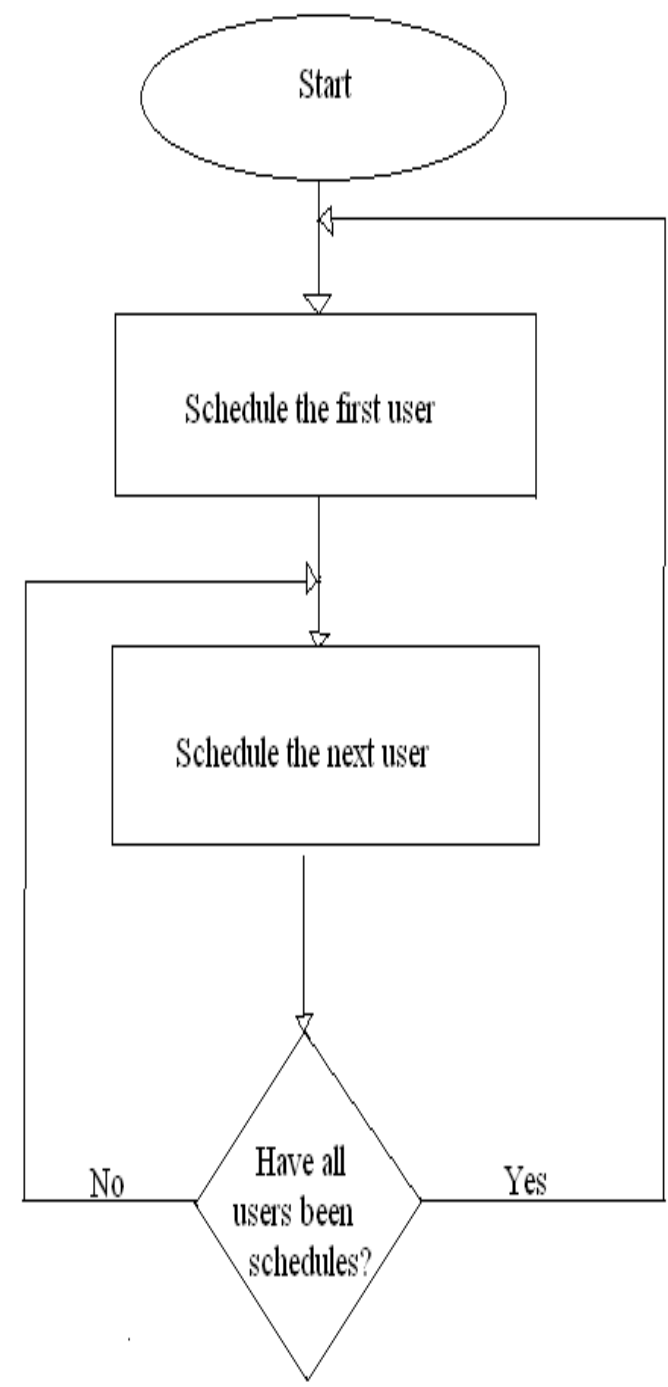

Fig.1. Round Robin Scheduling Flowchart

\section{B. Best Channel Quality Indicator ( Best CQI)}

CQI stands for Channel Quality Indicator. As the name implies, it is an indicator carrying the information on how good/bad the communication channel quality is. CQI is the information that UE sends to the network and practically it implies the following two: [19]

1. Current Communication Channel Quality is this-and-that.

2. UE wants to get the data with this-and-that transport block size, which in turn can be directly converted into throughput.

In LTE, there are 15 different CQI values ranging from 1 to 15 . Depending which value UE reports, network transmit data with different transport blocks size. If network gets high CQI value from UE, it transmits the data with larger transport block size and vice versa.

What if network sends a large transport block even though UE reports low CQI, it is highly probable that UE failed to decode it and UE send Negative Acknowledge (NACK) to network and the network have to retransmit it which in turn cause waste of radio resources.

What if UE report high CQI even when the real channel quality is poor? In this case, network would send a large transport block size according to the CQI value and it would become highly probable that UE failed to decode it and UE send NACK to network and the network have to retransmit it which in turn cause waste of radio resources [19]. The terminal reports the measured CQI to the BS by mapping the measured SNR according to Fig.2. 


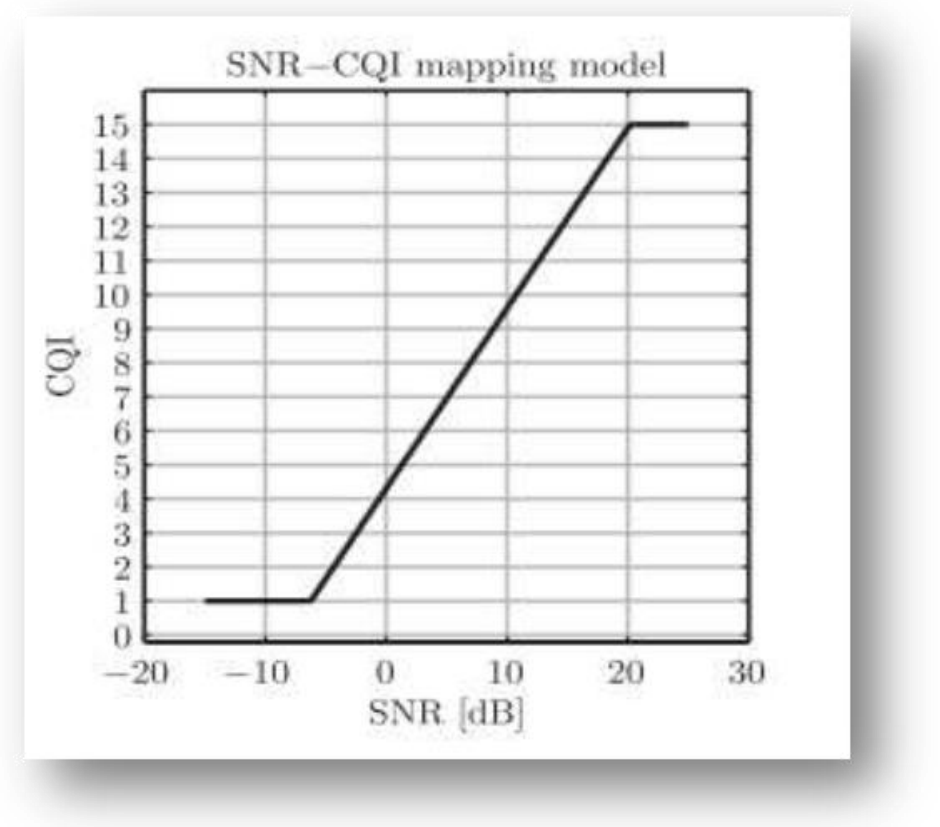

Fig.2. SNR-CQI Mapping Model

\section{Simulation Environment}

To calculate the throughput the following equations were used:

$N_{\text {coded hits }}=$ [modulation order $]$

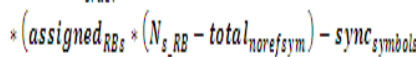

$$
\begin{aligned}
& \left.-\mathrm{CH}_{\text {sjmas }}\right)
\end{aligned}
$$

Where:

$N_{\text {codedits }}$ modulation onder $_{\text {. }}$ symbol respectively. assigned $d_{R B s}$ $N_{S_{-R B}}$ multiple by 7 OFDM total $l_{\text {no }}$ rafsym fifth OFDM sym sync $_{\text {symbols }}$

$\mathrm{CH}_{\text {ayng }}$

$N_{\text {data }_{\text {bits }}}=8 *$ round $\left(\frac{1}{8} * N_{\text {coded }_{\text {bits }}} * \frac{\left[\text { coding }_{\text {rate }_{\mathrm{Y}_{1024}}}\right]}{1024}\right)-24$

Where:

$\begin{array}{ll}N_{\text {data }_{\text {bits }}} & =\text { is the data to be sent, } \\ N_{\text {coded bits }} & =\text { is the data plus redundant bits }\end{array}$

$\operatorname{coding}_{\text {rate }_{\mathrm{K}_{1024}}}=$ is the Cyclic Redundancy Check $(\mathrm{CRC})=24$.

Throughput Value $=\frac{\left(\frac{N_{\text {databer }}}{\text { Tsubframe }}\right)}{10^{6}}$

Where:

Tsubframe $\quad=1 \mathrm{~ms}$

$=$ is the data plus redundant bits. case of short Cyclic Prefix (CP). $=$ Synchronization Symbols.

= Channel Symbols.

= QPSK, 16QAM, and 64QAM representing two, four, and six bits per modulation $=$ is the total number of resource block in each bandwidth

$=84$ is the number of symbols in one resource block (RB) which mean 12 subcarrier $=4$ are the reference symbols (reference signals) which are inserted in the first and 


\section{Conclusion}

The limited resources to transmit are an important fact to consider when the desire is to improve the speed of the transmissions. The different ways of sharing the available resources efficiently while trying not to interfere in high manner with the other transmissions is one of the problems of LTE. The scheduler is a very important element of the base station. It assigns the resource blocks to different users. In this project two scheduling algorithms were used; Best CQI and Round Robin scheduling. As the name implies, the Best CQI scheduling assigns the resource blocks to the user with the higher CQI. In Round Robin scheduling the terminals are assigned the resource blocks in turn (one after another).

These scheduling algorithms have been implemented in a MATLAB-based Link Level Simulator of the Vienna University. A comparative analysis between the scheduling algorithms based on their throughputs and fairness for different scenarios (different scheduling method and different number of users with different bandwidth) was carried out.

The results showed that the Best CQI scheduling maximizes the throughput by scheduling the user with the good channel quality and the Round Robin scheduling is fair since it equally schedules the terminals. It also showed that the throughput in the Best CQI algorithm was higher than throughput in the Round Robin (RR) algorithm this is due to that the fact that the resource blocks are allocated to the user with the highest value of CQI. The results also showed that as the number of users increase in both Round Robin and Best CQI the throughput decreases. It also showed that increasing the bandwidth result in increasing the throughput of the system in both Round Robin and Best CQI.

\section{References}

[1]. Tshiteya Dikamba,’Downlink Scheduling in 3GPP Long Term Evolution (LTE)”, Delft University of Technology, March 2011.

[2]. Jim Zyren, “Over view of the 3GPP Long Term Evolution Physical Layer", Free Scale, July 2007.

[3]. Werner Perndl, "Scheduling Algorithms for UMTS FDD Downlink", July 2001.

[4]. R. Basukala, "Impact of CQI Feedback Rate/Delay on Scheduling Video Streaming Services in LTE Downlink", University of Technology Sydney, December 2007.

[5]. Peter Skov, "Channel-Aware Frequency Domain Packet Scheduling for MBMS in LTE", Beijing University of Posts and Telecommunications, June 2009.

[6]. Centre Tecnològic de Telecomunicacions de Catalunya (CTTC), "LTE Simulator Documentation”, June 14, 2011.

[7]. Mustafa Ergen, "Mobile Broadband Including WiMAX and LTE", Springer, 2009.

[8]. DavidMart' in-Sacristan and other authors, "On theWay towards Fourth-GenerationMobile: 3GPP LTE and LTE-Advanced", EURASIP Journal onWireless Communications and Networking, June 2009

[9]. Harri Holma and Antti Toskala, "LTE for UMTS - OFDMA and SC-FDMA Based Radio Access", Wiley, 2009.

[10]. AndrasRacz and other authors, "Architecture and Protocol Support for Radio Resource Management (RRM)", Hungary, December 2007.

[11]. Sajid Hussein, "Dynamic Radio Resource Management in 3GPP LTE”, Blekinge Institute of Technology, January 2009.

[12]. Erik Dahlman and other authors, "Mobile Broadband Including WiMAX and LTE”, Oxford, 2008.

[13]. Erik Dahlman and other authors, "3G EvolutionHSPA and LTE for Mobile Broadband", Academic Press2008.

[14]. Erik Dahlman, "3G EVOLUTION: HSPA AND LTE FOR MOBILE BROADBAND”, 2008.

[15]. Rami J.Matarneh, "Self-AdjusmentTime in Round Robin Algorithm Depending on Burst Time of the Now Running Processes", Al-Isra Private University, 2009.

[16]. Albert Serra Pagès, "A Long Term Evolution Link Level Simulator", Universitat Politècnica de Catalunya, February, 2009 